\title{
Erratum: Kapitza stabilization of a repulsive Bose-Einstein condensate in an oscillating optical lattice [Phys. Rev. A 97, 023607 (2018)]
}

\author{
J. Martin, B. Georgeot, D. Guéry-Odelin, and D. L. Shepelyansky
}

(Received 8 March 2018; published 23 March 2018)

DOI: 10.1103/PhysRevA.97.039906

In the original paper, the characteristic oscillation frequency defined in the text, $\omega_{0}=2 \pi \sqrt{2 U_{0} /\left(m d^{2}\right)}$, differs by a factor $\sqrt{2}$ from the one used in the numerical simulations. Therefore, the definition of $\omega_{0}$ given in the original paper should be replaced by $\omega_{0}=2 \pi \sqrt{U_{0} /\left(m d^{2}\right)}$. As a consequence, the analytical estimates for the stability borders given in Eq. (4) of the original paper should read

$$
R_{0 \ell}>\sqrt{2} R_{i 0}, \quad R_{0 \ell}<0.45
$$

and, after Eq. (6) of the original paper, $R_{i 0 \text {,eff }}<R_{0 \ell} / \sqrt{2}$.

These two theoretical borders of Eq. (1) are shown by straight black dashed lines in the top right panel of Fig. 1 . We note that the lower border is in excellent agreement with the numerical data. The upper border is lower than the stability region centered around $R_{0 \ell} \approx 0.5$. The reason is that the Chirikov overlap criterion gives the border for a chaotic transition between resonances while the destruction of resonances takes place at higher values (e.g., in the Chirikov standard map, the primary resonance becomes unstable at $K \approx 2.5 S^{2}=4$ while the last invariant curve is destroyed at $K \approx 0.9716$ ). In our case with two primary resonances, the secondary resonance at $P=X=0$ becomes unstable at $R_{0 \ell} \approx 0.7$ and $R_{i 0}=0$, as it is shown in the Poincare sections in Fig. A for $R_{0 \ell}=0.65$ (fixed point $P=X=0$ is stable) and $R_{0 \ell}=0.7$ (fixed point $P=X=0$ is unstable) at $R_{i 0}=0$. The refined upper stability border $R_{0 \ell}=0.7$ is shown in the top right panel of Fig. 1 by the upper horizontal red dashed line.

The estimation of experimental parameters given in Sec. V of the original paper are slightly affected and should read: With $\omega_{\perp} \approx 2 \pi \times 200 \mathrm{~Hz}$ and a lattice spacing $d \approx 532 \mathrm{~nm}, \bar{g} \approx 0.003 N / \sqrt{s}$, we have $\bar{g} \approx 21$ for $N=10^{5}$ and a depth $s=200$, and $\bar{g} / Q \simeq 0.55$ for a BEC of typical size $20 \mu \mathrm{m}$.

These corrections leave all the conclusions unaffected.
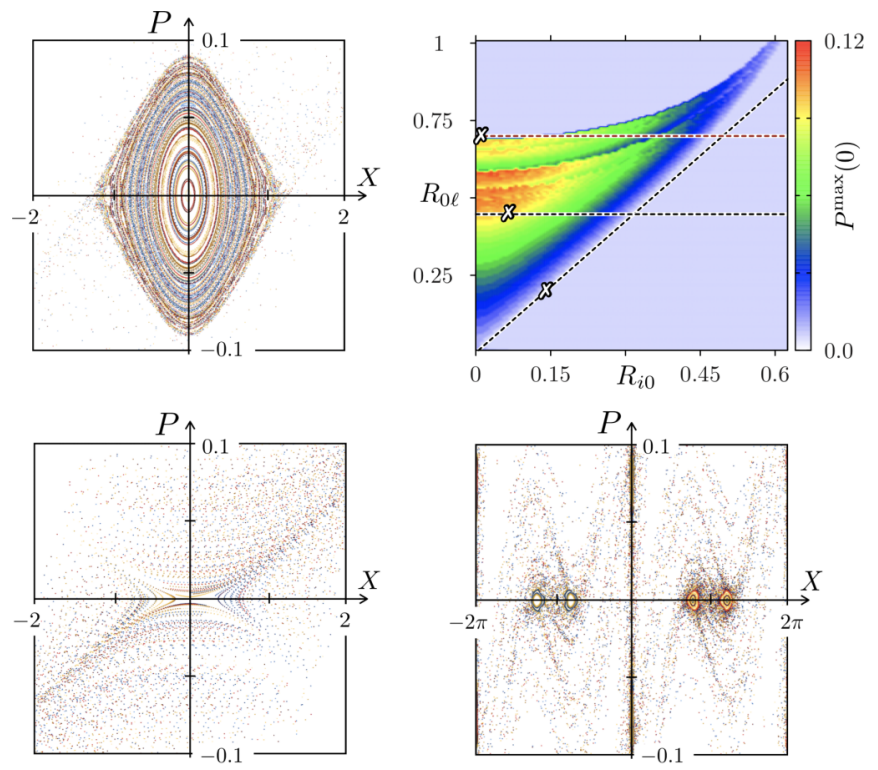

FIG. 1. Poincaré sections formed by a few thousand trajectories with random initial conditions $(X(0), P(0)) \in[-2 \xi: 2 \xi] \times[-0.1: 0.1]$ ( $\xi=1$ or $\pi$ ) propagated during a timespan $\Delta T=400$ for the frequency ratios $R_{i 0}=0.075$ and $R_{0 \ell}=0.45$ (top left), $R_{i 0}=0.15$ and $R_{0 \ell}=0.2$ (bottom left), and $R_{i 0}=0.02$ and $R_{0 \ell}=0.7$ (bottom right). (Top right) Stability region in the parameter space of frequency ratios. Color shows the largest initial momentum $P^{\max }(0)$ for which trajectories with initial conditions $(X(0), P(0))=(0, P(0))$ remain stable for time $T \in[0: 1000]$. Crosses mark parameters of the Poincaré sections; dashed black lines show theory (1), upper red/gray dashed line shows refined theory taking into account secondary resonances (see text). 

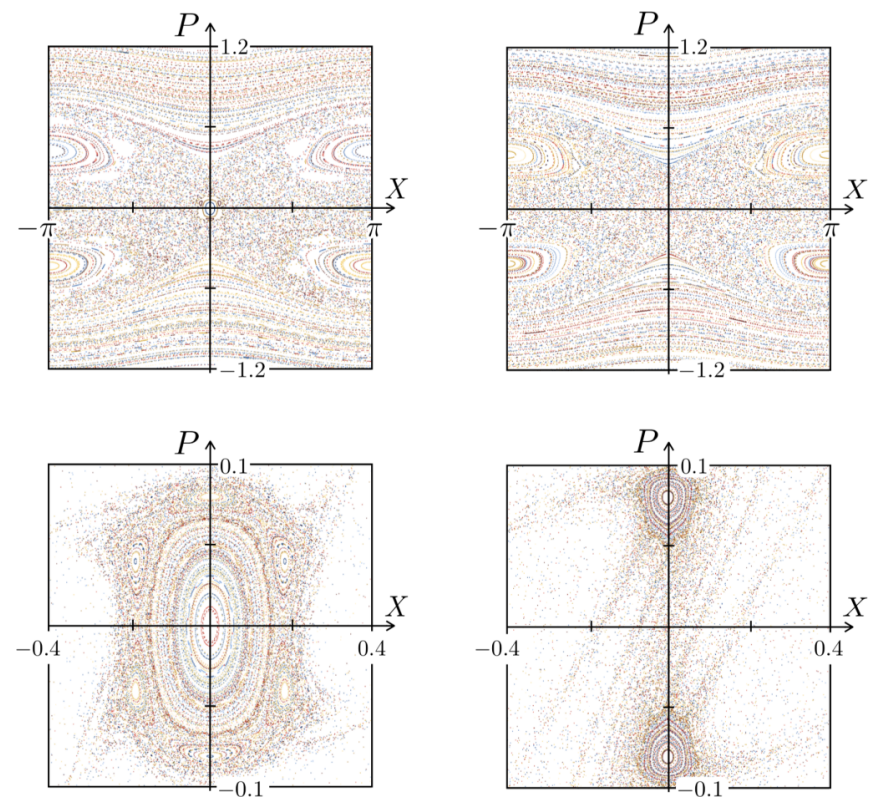

FIG. A. Poincaré sections for $R_{0 \ell}=0.65$ (left panels) and $R_{0 \ell}=0.7$ (right panels) at $R_{i 0}=0$ showing the loss of stability of the fixed point at $P=X=0$. In these plots, $X$ stands for $(X \bmod 2 \pi)-\pi$. 